\title{
On Conjugate -Gradient Algorithms
}

\author{
Shawki A.M. Abbas \\ Al-Nisour University College, Baghdad-Iraq. \\ Corresponding author: Shawki_45@ hotmail.com
}

\begin{abstract}
The aim of this paper is to recognize the attitude of the conjugate -Gradient Algorithms for solving linear systems $A x=b$ under the existence of rounding errors. The effect of matrix condition number of $\mathrm{A}$ on the relative error of the calculated series of approximations $\left\{x_{k}\right\}$ is analyzed. An especially appealing feature of the algorithm qualified is that error rating can be obtained very easily. Some examples are presented to support the theoretical results and to demonstrate the applicability and efficiency of the methods. The paper ends with some conclusions that sum up the finding of the study. The executed program for calculation is carried using "Matlb7".
\end{abstract}

[DOI: 10.22401/ANJS.21.4.10]

Keywords: Conjugate-gradient algorithms, Chebyshev methods, perturbed system, direct algorithms, Iterative algorithms, rounding errors.

\section{Introduction}

Let's assume we want to resolve the following order of linear equation " $\mathrm{Ax}=\mathrm{b}$ for the vector $\mathrm{x}$ where the known" nxn matrix $\mathrm{A}$ is symmetrical (i.e., $\mathrm{A}^{\mathrm{T}}=\mathrm{A}$ ), "positive definite (i.e., $\mathrm{x}^{\mathrm{T}} \mathrm{Ax}>0$ " for all non-zero vectors $\mathrm{x}$ in $\mathrm{R}^{\mathrm{n}}$ ), and $\mathrm{b}$ is known as well. We indicate the unique resolution of this order by $\mathrm{x}$. It is well determined that the cg. iterations impose "optimal complexity in a sense to be made precise." In accurate arithmetic it produces a series of perpendicular residual vectors $r_{k}=$ $A_{x_{k}}-b$, and the solution $\alpha=A^{-1} b$ is gained after mainly $\mathrm{n}$ steps [1,2,3].Many of these theoretical properties do not hold in the presence of "rounding errors". It is no longer correct that the calculated residual vectors are nearly perpendicular and that the $\mathrm{n}^{\text {th }}$ calculated vector $\mathrm{x}_{\mathrm{n}}$ is a rational approach to $\alpha$.

A especially "attractive feature of the algorithm described" is that error estimates ability can be obtained very easily. The objective of this paper is to realize the action of several cg Algorithms in the presence of rounding errors. We are mainly concerned with discussing how the matrix "condition number" $\mathrm{K}=\|A\|\left\|A^{-1}\right\|$, where $\|A\|$ donotes the spectral norm of $\mathrm{A}$, influences the relative error of the calculated series $\left\{x_{k}\right\}$.

We notice that immediate algorithms of functional advantage as well as much "iterative algorithms" with iterative refinement are fully behaved, i.e, $(\mathrm{A}+\delta \mathrm{A}) \mathrm{y}=\mathrm{b}$, where $\|\delta A\|$ is of order $\mathcal{T}\|A\|$ and $\mathcal{T}$ is the relative computer precision. Equivalently, the "residual vector $\mathrm{r}=\mathrm{A} y-\mathrm{b}$ " has a norm of order $\mathcal{T}\|A\|\|y\|$. When it not possible to be determined that an algorithm is fully behaved, it is occasionally likely to be a weak property, namely an algorithm is numerically steady, i.e, the relative error of a calculate $\mathrm{y}$ is of order $\mathcal{T}_{k}$ [4,5] for direct algorithms, and [7,10,11] "for iterative algorithms".

\section{Material and methods [6]}

We study "conjugate gradient algorithms" to work out uptight networks which are setup by a new modulate non monotonous planner study by Shi and Wany (2011). The use of non-monotonous planning can work out algorithm to cope with the status where the series of iterates runs into under most of a turned strict ravine, a common release in neural network practice procedure. Ours meant group of process guarantee enough descent, avoid there by the familiar inactive" restarts and it is globally convergent under" mid conditions. Our experimental results supply proof in order to suggest non monotonous conjugate gradient work out procedure are effective, outperforming classic procedure, provided extra settled, effective and credible study.

\subsection{Conjugate- gradient algorithms [6]}

The concept of what outcome can be expected form cg. Algorithms we record 
numeral exam. We examined [13] conjugate gradient algorithm for the numeral solution of special systems of linear equations "those whose matrix symmetric and positivedefinite". This method is often performed "as an iterative algorithm, usable to sparse systems that are "too large to be treated by direct application. We performed $\mathrm{j}(\mathrm{j} \geq n)$ iterative Steps finding the best possible approach $\mathrm{x}_{\mathrm{k}}, \mathrm{k} \leq j$, between all computed vectors. "Next we computed the relative error of $x_{k}$ and its residual vector. Define the number s such that"

$$
\frac{\left\|x_{k}-\alpha\right\|}{\left\|x_{k}\right\|}=\mathcal{T} k^{s}
$$

Let $\mathrm{s}=1$, which suggests the numerical stabilization of the algorithm. But, for maximum cases, s was around $\frac{3}{2}$ and "the residual vector had a spectral norm of order" $\mathcal{T}_{k}\|A\|\left\|x_{k}\right\|$.Thus, the algorithm is "neither well behaved nor numerically stable". A natural problem is to know why this is so and to request a cg. Algorithm which is numerically steady and well behaved.

To know why $s=\frac{3}{2}$, is called that the $\mathrm{cg}$. algorithms decrease the inaccuracy in the Anorm, $\left\|A^{1 / 2}\left(x_{k}-\alpha\right)\right\|$ thus it seems normal to size the inaccuracy by $\left\|A^{1 / 2}\left(x_{k}-\alpha\right)\right\|$ instead of by conjugate-gradient algorithms. $\| x_{k}-$ $\alpha \|$.Assume there occurs a numerically steady cg. Algorithm in the A-norm, i.e.,

$\left\|A^{1 / 2}\left(x_{k}-\alpha\right)\right\|=\mathrm{O}\left(\delta k\left\|A^{1 / 2} x_{k}\right\|\right)$

A reminder that the condition number of $\mathrm{A}$ in the A-norm concides "with the condition number of A in the spectral norm. Since"

$$
\left\|x_{k}-\alpha\right\| \leq\left\|A^{1 / 2}\right\|\left\|A^{1 / 2}\left(x_{k}-\alpha\right)\right\| \ldots
$$

Produce

$$
\left\|\left(\mathrm{x}_{\mathrm{k}}-\alpha\right)\right\|=O\left(\mathcal{T}_{\mathrm{K}}^{\frac{3}{2}}\left\|\mathrm{x}_{\mathrm{k}}\right\|\right)
$$

This clarifies why $s=\frac{3}{2}$ might be expected in (2.1.1).

We have not been successful in analyzing traditional cg. Algorithms, included that were suggested by [13]. In this paper we suggest a recent class of $\mathrm{cg}$. Algorithms and demonstrate that for these algorithms these occur a calculate vector $x_{k}$ such that
$\left\|A^{1 / 2}\left(x_{k}-\alpha\right)\right\| \leq C \mathcal{T}_{k}\left\|A^{1 / 2}\right\|\left\|x_{k}\right\|$

Where $\mathrm{C}$ is a constant of order at most n., we shall indicate this class of algorithms by $\phi$ where $\phi$ is a set. Note that K places linearly (2.1.4). In general, we cannot say that (2.1.4) means numerical stabilization of the cg. Algorithms is its "own"norm, since we have $\left\|A^{1 / 2}\right\|\left\|x_{k}\right\|$ instead of $\left\|A^{1 / 2} x_{k}\right\|$. However, if $\left\|A^{1 / 2}\right\|\left\|x_{k}\right\|$ is of $\mathrm{O}\left\|A^{1 / 2} x_{k}\right\|$, thereafter these cg. "Algorithms are numerically stable in the A-norm. For the residual vectors we are only able to prove that"

$\left\|\mathrm{r}_{\mathrm{k}}\right\| \leq C \mathcal{T}_{k}\|A\|\left\|x_{k}\right\|$

We examined one algorithm $\varphi$ form $\phi$. For extreme cases $\varphi$ matches a well- behaved algorithm, i.e., $\left\|r_{k}\right\|$ was of $\operatorname{rank} \mathcal{T}\|A\|\left\|x_{k}\right\|$ but, in few condition, $\left\|r_{k}\right\|$ was of rank $\mathcal{T}\|A\|\left\|x_{k}\right\|$. This evidence that (2.5) is sharp and some cg. Algorithms from $\phi$ are not well behaved.

Much iterative algorithms have this characteristic, i.e., "they are numerically stable but not well behaved. Examples". Include the chebychev, SOR, Richardson and Jacobi iterative algorithms $[10,11]$. However, it was shown in [7] "that any algorithm (direct or indirect) which calculate an approach y such as " $\|y-\alpha\| \leq q\|\alpha\|$ with $q \leq 1$ followed by iterative refinement in single precision becomes numerically stable, and if $\mathcal{T} k^{2}$ is of rank unity then it is also well behaved.

\subsection{Gradient and Conjugate Gradient Iterations}

We briefly conclude some basal properties of the gradient and conjugate - gradient iterations. We look it the solution of major linear system

$$
\mathrm{Ax}=\mathrm{b}
$$

Where $\mathrm{A}=\mathrm{A} *>0$ "is an $\mathrm{n} \times \mathrm{n}$ hermitian and positive definite matrix and $b$ is $a n \times$ 1 given vector. assume that the datum "absolute the matrix $\mathrm{A}$ is given by a step which calculate $\mathrm{y}=\mathrm{Ax}$ for a given $\mathrm{x}$. For large system A is commonly scattered, who declarations the valuation of $\mathrm{y}$ in time and storage proportional to $\mathrm{n}$. 
We resolve (2.2.1) iteratively by structuring a series $\left\{x_{k}\right\}$ approximate to the solution

$\alpha=A^{-1} b$. Let $\mathrm{B}=B^{*}>0$ be a matrix which replace which $\mathrm{A}: \mathrm{BA}=\mathrm{AB}$. For case one can set $\mathrm{B}=A^{p}$ for a real p. let $\|x\|_{B}=$ $\sqrt{\left(B_{x}, x\right)}=\left\|B^{1 / 2} x\right\|$, where $\|x\|=\sqrt{(x, x)}$ is the spectral norm.

We recall the introduction of the gradient iteration which build the sequence $\left\{x_{k}\right\}$ as follows. Lets $x_{0}$ be a given initial approach and

$x_{k+1}=x_{k}-c_{k} r_{k}, r_{k}=A_{x_{k}}-b$,

Where $c_{k}$ is election in such a way that the inaccuracy $\ell_{k+1}=\left\|x_{k+1}-\alpha\right\|_{B}$ is reduced, i.e., $\left\|x_{k+1}-\alpha\right\|_{B}=\inf \left\|x_{k}-c r_{k}\right\|_{B}$

This produce

$$
c_{k}=\frac{\left(r_{k}, \beta\left(x_{k}-\alpha\right)\right)}{\left(r_{k}, B r_{k}\right)}
$$

Retrieval that for $\mathrm{A}=\mathrm{B}$, the iteration (2.2.2),(2.2.3) is called the steepest - descent iteration. It has, in generic, very slow gathering and thus is not "recommended" in the numeral exercise. The conjugate -gradient iteration is so more effective. Next derivation of the cg. Iteration concentrates on its complexity optimality. Gaze a class of iterations for which the error formula secures the connection

$x_{k}-\alpha=w_{k}(A)\left(x_{0}-\alpha\right)$

Wherever $w_{k}$ is a polynomial of degree at most $\mathrm{k}$ and $w_{k}(0)=1$. A normal complication request is how to select the polynomials $w_{k}$. "Since we want to minimize the computational complexity (cost)", we seek $w_{k}$ such that inaccuracy $e_{k}=\left\|x_{k}-\propto\right\|_{B}$ is reduced. This wherewithal that the polynomials $w_{k}$ are the solution of the following problem:

$$
\left\|w_{k}(A)\left(x_{0}-\propto\right)\right\|_{B}=\inf \left\|p(A)\left(x_{0}-\propto\right)\right\|_{B}
$$

Wherever $w_{k}(0,1)$ is the class of ploynomials of degree at most $\mathrm{K}$ equalizes to unity in origin. The resolution of (2.2.5) is specific by the orthogonal polynomials defined as follows.

Let $x_{0}-\alpha=\sum_{j=1}^{m} c_{j} \xi_{j}$
Where $\xi_{j}$ is an eigenvector of A associated with the eignvalue $\lambda_{j}: \mathrm{A} \xi_{j}=\lambda_{j} \xi_{j}$

$\left\|\xi_{j}\right\|=1,0<\lambda_{1}<\lambda_{2}<\cdots<\lambda_{m}$, with $\mathrm{m} \leq \mathrm{n}$ and $\mathrm{cj} \neq 0$ for $\mathrm{j}=1,2, \ldots, \mathrm{m}$. memo that $\xi_{j}$ is as well an eigenvector of $\mathrm{B}: \mathrm{B} \xi_{j}=B_{j} \xi_{j}$ for $\mathrm{B}_{\mathrm{j}}>0, \mathrm{~J}=1,2, . ., \mathrm{m}$. know the inner product

$$
(f, g)=\sum_{j=1}^{m}|c j|^{2} \mathrm{~B}_{\mathrm{j}} \lambda_{\mathrm{j}} f\left(\lambda_{\mathrm{j}}\right) g \overline{\left(\lambda_{\mathrm{j}}\right)},
$$

Wherever $f$ and $g$ are function realize on the interval $\left[\lambda_{1}, \lambda_{\mathrm{m}}\right]$. The polynomials $w_{k}, w_{k}(0)=1$, "which minimize" (2.2.6) are the orthogonal polynomials with respect to inner production (2.2.7), i.e.,

$$
\left(w_{k}, w_{i}\right)=\sum_{J=1}^{m}[c j]^{2} B_{j} \lambda_{\mathrm{j}} w_{k}\left(\lambda_{\mathrm{j}}\right) \overline{w_{J}\left(\lambda_{\mathrm{J}}\right)}=0
$$

till $\mathrm{k} \neq i$, from the orthogonally of $w_{k}$ it follow up that they satisfy a three -term repetition formula. We select a different shape of the three-term repetition formula than usual in order to confirm the relationship between the $\mathrm{cg}$. iteration and the gradient one. This shape is realized as follows:

$W_{0}(\lambda) \equiv 1$,

$W_{1}(\lambda) \equiv 1-c_{0} \lambda$

$W_{k+1}(\lambda)=$

$\left\{W_{k}(\lambda)-c_{k} \lambda W_{k}(\lambda)-u_{k}\left\{W_{k-1}(\lambda)-W_{k}(\lambda)+\right.\right.$

$\left.\left.c_{k} \lambda W_{k}(\lambda)\right\}\right\} \mathrm{k} \geq 1$

Where

$c_{k}=\frac{\left(W_{k}, W_{k}\right)}{\left(\lambda W_{k}, W_{k}\right)}$

$\mu_{0}=0, \mu_{k}=$

$\frac{\left(W_{k}-c_{k} \lambda W_{k}, \frac{1}{\lambda}\left(W_{k-1}-W_{k}\right)+c_{k} W_{k}\right)}{\left(W_{k-1}-W_{k}+c_{k} \lambda W_{k}, \frac{1}{\lambda}\left(W_{k-1}-W_{k}\right)+c_{k} W_{k}\right)}, \mathrm{k} \geq 1$

For that we bring the three -term repetition formula for the series $\left\{x_{k}\right\}$,

$$
\begin{aligned}
& x_{k}-c_{k} r_{k}=z_{k}, \quad A_{x_{k}}-b=r_{k} \ldots \ldots \ldots . . . \\
& z_{k}-\mu_{k} y_{k}=x_{k+1}, \quad x_{k-1}-z_{k}=y_{k} \\
& u_{0}=0, u_{k}=\frac{\left(y_{k, B\left(z_{k}-\alpha\right)}\right)}{\left(y_{k}, B y_{k}\right)}, \mathrm{k} \geq 1
\end{aligned}
$$

\subsection{Round off Error Analysis of Gradient Algorithms}

We will presentation the round off-error test of cg. Algorithms relationship to $\phi$ ability 
be firstly established on the round off -error analysis of the gradient algorithms to be thoughtful in this division. "Therefore in this section we analyze gradient algorithms in the presence of rounding errors". We focus our attention the steepest -descent algorithm $(\mathrm{A}=\mathrm{B})$ and recall the identical outcome for the gradient algorithms with $\mathrm{B}=\mathrm{I}$ or $\mathrm{A}^{2}=\mathrm{B}$

We gaze a steepest descent algorithm in floating-point binary arithmetical (f $\ell$ ) with the relative computer precision $\mathcal{T}=2^{-\mathrm{t}}$, "where $\mathrm{t}$ is the number of mantissa bits". To extend further rating we shall use the relationship, which is known as follows. Let $\mathrm{f}$ and $\mathrm{h}$ be two scalar functions defined on $\left[0, \mathcal{T}_{0}\right]$. By

$\mathrm{f}(\mathcal{T})=\mathrm{T}(\mathcal{T})$

We denote that there occur a constant $\mathrm{c}$ such that $\mathrm{f}(\mathcal{T})=\mathrm{h}(\mathcal{T})[1+c(\mathcal{T})]$, where $|c(\mathcal{T})| \leq c \mathcal{T}$ for $0 \leq \mathcal{T} \leq \mathcal{T}_{0}$. By

We pass

$$
\mathrm{f}(\mathcal{T}) \underset{1}{ } h(\mathcal{T})
$$

$$
\mathrm{f}(\mathcal{T}) \leq h(\mathcal{T}) \text { or } \mathrm{f}(\mathcal{T})=h(\mathcal{T})
$$

The relation $\leq$ enable as to eliminate the terms of rank $\mathcal{T}^{2}$ in the existence of the term of order $\mathcal{T}$. allow $r_{k}$ and $\mathrm{x}_{k}$ denote the vectors calculate in $\mathrm{f} \ell$ by an algorithms. We suppose that

$\mathrm{f} \ell\left(A_{\mathrm{x}_{k}}-b\right)=r_{k}$

Lemma 2.3.1:[2] Suppose that $\left\|\mathrm{r}_{\mathrm{k}}\right\|>$ $\mathcal{T}\|A\|\left\|x_{k}\right\| C_{1} \forall k$. Then series $\left\{x_{k}\right\}$ is calculated by algorithm accept the following inaccuracy formula:

$\sqrt{e_{k}^{2}-c_{k}^{*}\left\|r_{k}^{*}\right\|^{2}}+\left\|A^{1 / 2}\right\|\left\|x_{k}\right\|+$ $\mathcal{T} c_{k}^{*}\left\{\left\|A^{3 / 2}\right\|\left\|x_{k}\right\| 5 C_{1}+\|A\| e_{k}\left(C_{1}+2 C_{2}+\right.\right.$

8) $\} \geq e_{k+1}$

Where $C_{1}, C_{2}$ constants

Where $r_{k}^{*}=\left(r_{k}^{*}, r_{k}^{*}\right) /\left(r_{k}^{*}, A r_{k}^{*}\right)=A_{x_{k}}-b, c_{k}^{*}$

Lemma 2.3.2: [2] Show how the inaccuracy $e_{k+1}$ relies on the theoretical and "rounding errors". It is good to notice that the bound-on the rounding error raise which $c_{k}^{*}$, whilst the bounded on the theoretical error decreases with increasing $c_{k}^{*}$.

We need to discovery the limiting possession of the series $\left\{e_{k}\right\}$ which satisfies (2.3.2). to attain this we use the next lemma.
Lemma2.3.3: [2] allow

$\sqrt{e_{k}^{2}-c_{k}^{*}\left\|r_{k}^{*}\right\|^{2}}+a k+c_{k}^{*} b_{k}+c_{k}^{*} e_{k} d \geq e_{k+1}$ Till specified nonnegative sequence " $\left\{a_{k}\right\},\left\{b_{k}\right\}$ and a constant $d$ such that" $2 \mathrm{~d}\left\|A^{-1}\right\|<1$. Thereafter

$\underset{\mathrm{k}}{\overline{\operatorname{llm}} e_{k}} \leq \underset{\mathrm{k}}{3 k} \frac{\overline{l l m} \frac{b k}{\|A\|}+\overline{l \iota m} a_{k}}{1-\frac{2 d k}{\|A\|}} \mathrm{k}$

Theorm 2.3.4[2] if $\beta=2 \mathcal{d f}_{k}\left(C_{1}+2 C_{2}+8\right)<$ 1 , then algorithm computes the seq $\left\{\mathrm{x}_{k}\right\}$ such that

$\overline{\lim }\left\|A^{1 / 2}\left(\mathrm{x}_{k}-\propto\right)\right\| \leq$

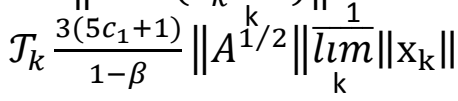

Corollary 2.3.5: [2] brief the numerical estate of the "steepest-descent algorithm. It shows that the algorithm may be neither well behaved nor numerically stable".

From the above lemmas we at once seal the asymptotic attitude of the series $\left\{x_{n}\right\}$ calculated by algorithm (2.3.1).

\section{Samples analysis}

"Numerical tests confirm that the residual vectors sometimes depend" on $\mathcal{T}_{k}$. This means that algorithms are not well behaved. However, if $\left\|A_{x_{k}}-b\right\| \cong\left\|A^{1 / 2}\left(x_{k}-\alpha\right)\right\| /$ $\left\|A^{-1 / 2}\right\|$, then the residual vectors $r_{k}^{*}$. rely at worst on $\mathcal{T}_{k}^{1 / 2}$.

"Numerical stability and/or the wellbehaved property may be achieved by the use of iterative refinement" even if the residual are calculate in single accuracy. From theorems [7] it follows up that algorithm with iterative refinement in single accuracy is numerically stable whenever $1>\mathcal{T} k^{3 / 2} C$, and it is well behaved where $\mathcal{T} k^{2}$ is at maximum of rank unity.

\section{Result and Discussion}

The researchers progressing a new group of conjugate gradient process for unaffected optimization problems. A new no monotonous line search style is suggested to warranty the global concourse of those conjugate gradient methods down about reasonable case in specific, Lu-Storey \& Polak-Ribiere conjugate gradient process are specific situation of the new class of conjugate gradient methods. 
By considering the local lipschitz constant [24] of the derivative of the objective functions, we can discover and appropriate step size and basically reduces the function valuation at every iteration.

"Numerical results show that these new conjugate gradient methods are" active in reducing large -scale non -convex non quadratic functions.

\subsection{Numerical Examples Example 4.1.1 [10]}

Let $x_{k}$ and $x_{k-1}$ be the calculate vectors and $r_{k}, r_{k-1}$ the corresponding residual vectors.

Let $f \ell\left(A_{r_{k}}\right)=v_{k}$ be the calculate vector which is used for the calculation of $c_{k}$.

We suggest the following algorithm for the calculation of $\mu_{k}$. Let

$f \ell\left(\left(y_{k}, r_{k}-c_{k} v_{k}\right)\right)=w_{1}$,

$f \ell\left(\left(y_{k}, r_{k-1}-r_{k}+c_{k} v_{k}\right)\right)=w_{2}$.

So the counting of $w_{1}$ and $w_{2}$ does not require moreover matrix- vector multiplication. cyclic a portion of the analysis before it is likely to show that

$w_{1}=\left(y_{k}, A\left(z_{k}-\alpha\right)+\delta w_{1}\right.$,

$\left|\delta w_{1}\right| \leq \delta\|A\|\left\|y_{k}\right\|\left\|x_{k}\right\| C_{3}$,

$w_{2}=\left(y_{k}, A y_{k}+\delta w_{2},\left|\delta w_{2}\right| \leq\right.$

$\delta\|A\|\left\|y_{k}\right\|\left\|x_{k}\right\| C_{4}$,

Where $C_{3} \cong C_{1}+1$ and $C_{4} \cong 2 C_{1}+1$. From this we get

$\frac{w_{1}}{w_{2}}=\mu_{k}\left(1+\delta \mu_{k}\right)$

$\left\|\delta \mu_{k}\right\| \leq \mathcal{T}\|A\|\left\|y_{k}\right\|\left\|x_{k}\right\|\left(\frac{C_{3}}{\left|w_{1}\right|}+\frac{C_{4}}{\left|w_{2}\right|}\right)$

This suggest the following algorithm for the calculation of $\tilde{\mu}_{k}$,

$\tilde{\mu}_{k}=\left\{\begin{array}{cc}\frac{w_{1}}{w_{2}} & \text { if } \mathcal{T}\|A\|\left\|y_{k}\right\|\left\|x_{k}\right\|\left(\frac{C_{3}}{\left|w_{1}\right|}+\frac{C_{4}}{\left|w_{2}\right|}\right)<1 \\ 0 & \text { otherwise }\end{array}\right.$

Hence, $\quad \bar{\mu}_{k}=\mu_{k}\left(1+\delta \mu_{k}\right),\left|\delta \mu_{k}\right| \leq 1$ is content. Reminder that $\tilde{\mu}_{k}=0$ means in order to $z_{k}=x_{k+1}=f l\left(x_{k}-c_{k} r_{k}\right)$ is gained by one step of the steepest-descent algorithm. "This can be interpreted as the initialization of the cg algorithm from the" vector $x_{k}$.

It may also be observed while vector $z_{k}$ and $y_{k}$ need not be stocked. One step of the algorithm can be perfect having five vectors $x_{k}, x_{k-1}, r_{k}, r_{k-1}$ and $v_{k}=A_{r_{k}}$ in storage and use two matrix-vector multiplications.

We have complete much numerical workout employ this algorithm. In almost condition the algorithm was well behaved in the spectral norm. Much, in a few situation (about 5 present) numerical tests experimentally certain the sharpness of the error bounds in Corollory (2.3.5).

Example 4.1.2: (Encyclopedia) gaze the linear system $A x=b$ given by

$\mathrm{Ax}=\left[\begin{array}{ll}4 & 1 \\ 1 & 3\end{array}\right]\left[\begin{array}{l}x_{1} \\ x_{2}\end{array}\right]=\left[\begin{array}{l}1 \\ 2\end{array}\right]$

We will execute the two steps of the conjugate gradient method beginning with the initial rating $x_{0}=\left[\begin{array}{l}2 \\ 1\end{array}\right]$ "in order to find approximate solution to the system". resolution to assurance the accurate solution is:

$\mathrm{X}=\left[\begin{array}{c}\frac{1}{11} \\ \frac{7}{11}\end{array}\right] \approx\left[\begin{array}{l}0.0909 \\ 0.6364\end{array}\right]$

Ours first stage is to enumerate the residue vector $r_{0}$ related with $x_{0}$. This residue is calculate for the form: $b-A x_{0}=r_{0}$ is equal to:

$r_{0}=\left[\begin{array}{l}1 \\ 2\end{array}\right]-\left[\begin{array}{ll}4 & 1 \\ 1 & 3\end{array}\right]\left[\begin{array}{l}2 \\ 1\end{array}\right]=\left[\begin{array}{l}-8 \\ -3\end{array}\right]$

Since that is the first iteration we would used the residue "vector $r_{0}$ as our initial search direction $\mathrm{P}_{0}$ ": the style of chosen $p_{k}$ would alteration in otherwise iterations.

We now count the scalar $a_{0}$ employ the related

$a_{0}=\frac{r_{0}^{T} r_{0}}{p_{0}^{T} A p_{0}}=\frac{\left[\begin{array}{ll}-8 & -3\end{array}\right]\left[\begin{array}{l}-8 \\ -3\end{array}\right]}{\left[\begin{array}{ll}-8 & -3\end{array}\right]\left[\begin{array}{ll}4 & 1 \\ 1 & 3\end{array}\right]\left[\begin{array}{l}-8 \\ -3\end{array}\right]}=\frac{73}{331}$

We can now calculate $\mathrm{x}_{1}$ using the form:

$\mathrm{x}_{1}=\mathrm{x}_{0}+a_{0} p_{0}=\left[\begin{array}{l}2 \\ 1\end{array}\right]+\frac{73}{331}\left[\begin{array}{l}-8 \\ -3\end{array}\right]=\left[\begin{array}{l}0.2356 \\ 0.3384\end{array}\right]$

That outcome complete the first iteration, the outcome existence an "amended" convergent solution to the system, $\mathrm{x}_{1}$. We may now proceed on and calculate the following residue vector $r_{1}$ using the formula. 
$r_{1}=r_{0}-a_{0} A p_{0}=\left[\begin{array}{l}-8 \\ -3\end{array}\right]-\frac{73}{331}\left[\begin{array}{ll}4 & 1 \\ 1 & 3\end{array}\right]\left[\begin{array}{l}-8 \\ -3\end{array}\right]=$ $\left[\begin{array}{c}-0.2810 \\ 0.7492\end{array}\right]$

Our next step in the method is to calculate $\beta_{0}$ that will eventually be used to decide the next discussion direction $p_{1}$.

$\beta=\frac{r_{1}^{T} r_{1}}{r_{0}^{T} r_{0}}=\frac{\left[\begin{array}{ll}-0.2810 & 0.7992\end{array}\right]\left[\begin{array}{c}-0.2810 \\ 0.7492\end{array}\right]}{\left[\begin{array}{ll}-8 & -3\end{array}\right]\left[\begin{array}{l}-8 \\ -3\end{array}\right]}=0.0088$

Now using $\beta_{0}$. We can enumerate the next discussion direction $p_{1}$ using the form:

$p_{1}=r_{1}-\beta_{0} p_{0}=\left[\begin{array}{c}-0.2810 \\ 0.7492\end{array}\right]+0.0088\left[\begin{array}{l}-8 \\ -3\end{array}\right]=$

$\left[\begin{array}{c}-0.3511 \\ 0.7229\end{array}\right]$

We now enumerate the scalar $a_{1}$ using our new gained $p_{1}$

Using the same procedure as that used for $a_{0}$

$a_{1}=\frac{r_{1}^{T} r_{1}}{p_{1}^{T} A p_{1}}=\frac{\left[\begin{array}{ll}-0.2810 & 0.7492\end{array}\right]\left[\begin{array}{c}-0.2810 \\ 0.7492\end{array}\right]}{\left[\begin{array}{ll}-0.2810 & 0.7492\end{array}\right]\left[\begin{array}{cc}4 & 1 \\ 1 & 3\end{array}\right]\left[\begin{array}{c}-0.3511 \\ 0.7229\end{array}\right]}=$

0.4122

Lastly, we discovery that $\mathrm{x}_{2}$ utilizes the himself process as that applied to discovery $x_{1}$

$\mathrm{x}_{2}=\mathrm{x}_{1}+a_{1} p_{1}=$

$\left[\begin{array}{l}0.2356 \\ 0.3384\end{array}\right]+0.4122\left[\begin{array}{c}-0.3511 \\ 0.7229\end{array}\right]=\left[\begin{array}{l}0.0909 \\ 0.6364\end{array}\right]$

The outcome, $\mathrm{x}_{2}$ is a "better" approach to the system solution than $\mathrm{x}_{1}$ and $\mathrm{x}_{0}$.If accurate arithmetical were to be applied in this example instead of limited-accuracy, then the precise solution would in theory be to attend next $n=2$ repetition ( $\mathrm{n}$ being the order of the system).

\section{Example 4.1.3, [18]}

As a numerical example we gaze the wellknown five-point difference approach of the Poisson equation with homogeneous "Dirichlet boundary conditions using an equidistant mesh of mesh width 1 in a rectangle having side lengths 12 and 6". The system of difference equations" depends of $n=55$ linear equations, the associated coefficient matrix $\mathrm{A}$ is a band matrix of band width 11. In addition", $A$ is an M-matrix so that $\mathrm{A}^{-1}, \mathrm{U}^{-1}$ are nonnegative. Hence the condition numbers $\sigma_{i}^{D}, \sigma_{i}^{R}$ of the unknown's $x_{i}$ can be specific simply as solutions of the linear systems
$A \sigma^{D}=\tau^{D}$

$A \sigma^{0}=\tau^{0}, U \sigma^{1}=X, \quad \sigma^{R}=\sigma^{0}+\sigma^{1}$,

Where

$X_{j}=\sum_{k=j+1}^{n}\left|\bar{z}_{j}^{k}\right| \varepsilon_{j k}^{-}+\sum_{k=j}^{n}\left|\bar{u}_{j k} \bar{x}_{k}\right|(j=1, \ldots, n)$.

"The matrix A and the right-hand side $\mathrm{y}=(1, \ldots, 1)$ of the system of difference equations were first multiplied by 0.9973 and then rounded symmetrically (B15) or truncated (C15), respectively, to 15 binary digits. Next, the solution vector of the linear system was computed. The arithmetic operations of the floating-point arithmetic were carried out in the form $\mathrm{fl}_{\mathrm{N}}(\mathrm{a} \circ \mathrm{b})$ " wherever $\mathrm{fl}_{\mathrm{N}}$ denotes symmetrical round $(\mathrm{BN})$ "or chopping $(\mathrm{CN})$ to $\mathrm{N}$ binary digits and. $\mathrm{a} \circ \mathrm{b}$ for $\mathrm{o}=+,-, \mathrm{x}, /$ is the result of about 9 decimal digits floating-point arithmetic of the desk top computer, the condition numbers were computed in the builtin floating-point arithmetic of the computer using the weights" $\varepsilon_{r i k}^{-}, \varepsilon_{j k}^{-}$specified by

$\bar{m}_{i t}=0: \varepsilon_{t i}^{\prime}=0$

$\bar{m}_{i t}=0$ or $u_{t k}=0: \varepsilon_{t i k}^{x}=0$

$a_{i k}{ }^{-t}=0$ or $\bar{m}_{i t}=0$ or

$\bar{u}_{i k}=0: \bar{e}_{t i k}=0$.

And in back substitution

$\bar{u}_{i k}=0$ or $\bar{x}_{k}=0: \varepsilon_{j k}^{x}=0 . z_{j}^{-k+1}=0$ or

$\mathrm{f} 1\left(\bar{u}_{i j_{k}}, \bar{x}_{j_{k}}\right)=0: \varepsilon_{j k}^{\bar{z}}=0$

and $\varepsilon_{r i k}^{-}=\varepsilon_{j k}^{-}=1$ else.

Table (1) display a chain of relative data and rounding condition numbers the solutions $\mathrm{x}_{\mathrm{i}}$ and the connected residual condition numbers together with the error and residual percentages

$P i \%=100 \frac{\left|P \mathrm{x}_{\mathrm{i}}\right|}{\rho_{i}^{R} \eta_{R}}, \quad \varrho_{i} \%=\frac{\left|(A \bar{x}-y)_{i}\right|}{\tau_{i}^{R} \eta_{R}}$

The "floating-point accuracy constant has the value"

$(B N): \eta_{R}=2^{-N},(C N): \eta_{R}=2^{-N+1}$

It is clear from Table (1) that the error proportion of the floating-point arithmetic (C15) are safely greater than those of the arithmetic (B 15) for A is an M-matrix and the right-hand side $y \geq 0$ This reality has been explained before. The maximal relative error of the computed solutions is overestimated by a factor of about 11 and 8 , respectively. 
Table (1)

Five-point difference approximation of the Poisson equation, condition numbers of the solutions $x_{i}$, error and residual percentages.

\begin{tabular}{|c|c|c|c|c|c|c|c|c|}
\hline $\begin{array}{c}\text { Float.pt } \\
\text { i } \\
\end{array}$ & $\begin{array}{c}\text { Arithmetic } \\
\rho_{i}^{D} \\
\end{array}$ & $\rho_{i}^{R}$ & $\begin{array}{c}\text { (B15) } \\
P_{i} \% \\
\end{array}$ & $\begin{array}{c}(\mathrm{C} 15) \\
P_{i} \% \\
\end{array}$ & $\tau_{i}^{D}$ & $\tau_{i}^{R}$ & $\begin{array}{c}(\mathrm{B} 15) \\
\varrho_{i} \% \\
\end{array}$ & $\begin{array}{c}(\mathrm{C} 15) \\
\varrho_{i} \% \\
\end{array}$ \\
\hline 1 & 16 & 70 & 1 & 10 & 8 & 14 & 14 & 35 \\
\hline 2 & 18 & 81 & 1 & 10 & 13 & 37 & 16 & 21 \\
\hline 3 & 19 & 84 & 0 & 9 & 14 & 50 & 16 & 26 \\
\hline 4 & 18 & 82 & 1 & 9 & 13 & 54 & 4 & 20 \\
\hline 5 & 16 & 71 & 1 & 8 & 8 & 43 & 5 & 12 \\
\hline 8 & 22 & 105 & 1 & 6 & 23 & 169 & 5 & 1 \\
\hline 18 & 25 & 123 & 2 & 7 & 31 & 242 & 12 & 5 \\
\hline 26 & 24 & 118 & 3 & 10 & 18 & 137 & 15 & 12 \\
\hline 27 & 25 & 126 & 2 & 9 & 29 & 225 & 0 & 6 \\
\hline 28 & 26 & 128 & 2 & 10 & 33 & 261 & 3 & 11 \\
\hline 29 & 25 & 125 & 3 & 9 & 29 & 242 & 12 & 11 \\
\hline 30 & 24 & 117 & 3 & 8 & 18 & 167 & 2 & 1 \\
\hline 38 & 25 & 123 & 5 & 9 & 31 & 249 & 0 & 2 \\
\hline 48 & 22 & 106 & 8 & 11 & 23 & 192 & 6 & 9 \\
\hline 51 & 16 & 81 & 6 & 12 & 8 & 72 & 6 & 11 \\
\hline 52 & 18 & 90 & 5 & 12 & 13 & 108 & 7 & 6 \\
\hline 53 & 19 & 92 & 8 & 12 & 14 & 116 & 5 & 7 \\
\hline 54 & 18 & 88 & 9 & 12 & 13 & 100 & 10 & 8 \\
\hline 55 & 16 & 78 & 9 & 13 & 8 & 36 & 3 & 13 \\
\hline
\end{tabular}

Furthermore see Ex [2,427-428].

\section{Conclusion}

We have shown in this paper that the relative error of computed vector $\mathrm{x}_{k}$ by $\mathrm{cg}$. Algorithm at worst on $\mathcal{T}_{k}^{3 / 2}$ for the relative computer precision $\mathcal{T}$. Moreover, for numerous functional situation the desired precision is great than $\mathcal{T}_{k}^{3 / 2}$. This is a fully favorable outcome.

As we mentioned previously, our process is not achieved in analyzing traditional cg. Algorithms, but we proved that at fully several of them has identical numerical ownership.

We watch experimentally that the calculated series first approximate the exact solution of the system $\propto$ at least as quick as the Chebyshev repetition.

Moreover, in many condition the error $\left\|\mathrm{x}_{\mathrm{k}}-\propto\right\|_{A}$ is safely minimal than the over restricted.

\section{Recommendation}

A modern group of adaptive algorithms is suggested to be found "on a uniformly" spread strings arrangement by affect its gravity vector to affixed combine symmetric form. The process is used to the well-known reference signal based (RSB) and it will be former and the linearly forced lower variance (LCMV) ray previous as two application models. The action of the extra bonds is equal to gather a second step in the derived adaptive algorithm. Only, a difference grow for the RSB situation ago no "direction-of-arrival "(DOA) notification of the required indicative is obtainable, who leads to a two -stage frame for combine the enjoined bands. Match to the classic algorithms. The suggest one's ability obtain a faster gathering speed and higher stable state produce signal to- choas-plus-noise ratio, give the himself step size.

\section{Reference}

[1] Engeli M., Rute'shauser Th., Ginsburg H. and stiefel E., "Refined iteartive Methods for computation of the solution and the Eigenvalues of self- Adjoint Boundary value problems", Birkhauser, Stuttgart, 1959.

[2] Hestenes M.R., and Stiefel E., "Methods of conjugate gradients for solving linear 
systems", J. Res. Nat, Bur. Standards, 59, 409- 436, 1952.

[3] Stiefel E., "Kernal polynomials in Linear algebra and their numerical applications", NBS Appl Math 49, 1-22, 1958.

[4] Wilkinson J. M., "Roanding Errors in Algebraic processes prentice- hall, Englewood cliffs", N.J, 1963.

[5] Wilkinson J. M., "The Algebraic Eigen value problem, Clarendon, oxford", 1965.

[6] averiel M.,"Nonlinear programming: Analysis and methods Dover Publishing". ISBN.O- 486-43227-0, 2003.

[7] Janowski M. \& wozniaki H., "itertative refinement implies numerical stability, Nordisk Tidskr, informations behanding" (Bit)17, 303-3011, 1977.

[8] Massachusetts institute of Technology, "Introduction to linear algebra, Fifth edition, publication" May, Gilbert strang: (Wellesley-Cambridge press\& SIAM (Book order from), 2016.

[9] Lipsohatz symour order Lipson Mark "Schaum's outlines": Linear algebra Tata McGraw- Hill edition, Delhi, 69- 80,2001.

[10] Wozniakowiski H., "Roundoff error analysis of iterations for large linear system", Namor. Math, 301, 301-314, 1978.

[11] Wozniakowiski H., "Numerical stability of chebyshev method for the solution of the large linear systems", Namer. Math 28, 191-209, 1977.

[12] Peng C.C. \& Magoulas G.D., "Anew class of nonmontone conjugate gradient traning, published in journal applied Mathematics and computation", 266(c), under Elsevier science inc. Nework, USA, 404-413, 2015.

[13] Concus P. Golub G.H. and O.Leary D.P., "A generalized conjugate gradient method for the numerical solution of elliptic partial differential equations, in sparse Matrix computations" (J.R.Bunch and D.J Rose, Eds.) Academic Nework, 309-332, 1976.

[14] Zhen-Jun shi. \& Jinhua Guo. "Anew family of conjugate gradient methods, Journal of computational and applied Mathematics", 224 (issue1) under an Elsevier user License, 444-457, 2009.
[15] Fox L. and parker I.B., "Polynomial in numerical analysis, oxford university press", 1968.

[16] Wilkinson J.H., "Rounding errors in algebraic processes". Prentice Hall, Englewood cliffs, 1963.

[17] Stummel F., "Perturbation theory for evaluation algorithms of arithematic expressions Math. Comp 37, no, 156, 435473, 1981.

[18] Stummel F., "Forward error analysis of Gaussion elimination part1": 46( 3), 365395, 1974.

[19] Nai- kuan Tsao, "Some posterior error bounds in flooting point computations": J.ACM21, 1974.

[20] Fangmin chen. \& Hans Hallez and steven staelens, "Influence of skull conductivity perturbation on EEG dipole source analysis", medical physics,37 (8), 44754484, 2010.

[21] Szego G. "Toeplitz forms and their application (books) google.com.U. Grenander, 2001.

[22] Kielbasinski A., "An introduction algorithm with corrections and some of its applications" in polish, Mat Stosowana 1, 23-41, 1973.

[23] Wieladek R. "Roundoff error analysis of the minimeal residual method in publication of the Geophysics", polish Academy of sciences, PWn 58 (7), 39163922, 1978.

[24] Lizhang wei Liv., Richard J. Langley, "Aclass of consirained Adaptive Bcamforming algorthms Baed on uniform Lineararrays", 2010. 